\title{
ENSAIOS EM VIGAS DE ALVENARIA SUBMETIDAS À FLEXÃO SIMPLES
}

\author{
Tests in Masonry Beams Subjected to Flexure
}

\author{
Maria das Graças Duarte de Oliveira ${ }^{1}$, Edgar Bacarjii ${ }^{2}$, Gilson Natal Guimarães ${ }^{3}$
}

Recebido em 23 de fevereiro de 2017; recebido para revisão em 20 de junho de 2017; aceito em 08 de julho de

2017; disponivel on-line em 25 de agosto de 2017.

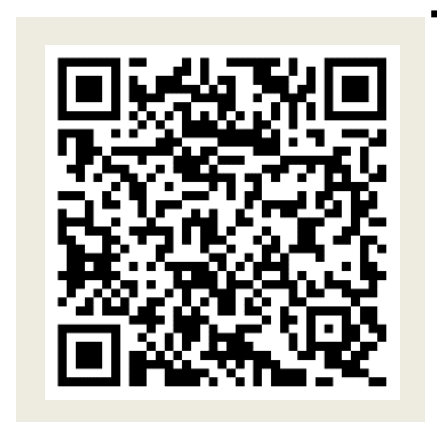

PALAVRAS CHAVE:

Ensaios;

Vigas;

Alvenaria estrutural;

Flexão simples;

Cisalhamento.

\section{KEYWORDS:}

Tests;

Beams,

Structural masonry;

Flexure;

Shear.

RESUMO: Foi realizado um estudo experimental de vigas de alvenaria de concreto submetidas à flexão simples e as variáveis usadas foram a altura da seção transversal e a distribuição das barras da armadura longitudinal. Foram confeccionadas nove vigas com blocos e canaletas de concreto de resistência característica a compressão de 13 MPa. A altura da seção transversal foi definida pela quantidade de fiadas de blocos: uma, duas e três fiadas. As barras de aço da armadura longitudinal foram distribuídas na seção transversal, mantendo constante a taxa de armadura. Foram moldadas três vigas apenas com canaletas e sem estribos, pois seu uso é muito comum na região de Goiânia, GO. Os resultados obtidos foram confrontados com valores encontrados utilizando as recomendações de dimensionamento da norma NBR15961-1 (2011). De modo geral, as vigas com estribos e com armadura longitudinal distribuídas nas diversas fiadas tiveram desempenho melhor, sendo estas mais dúcteis e mais seguras que as vigas apenas com canaletas e sem estribos. Essas vigas com estribos romperam por flexão e as cargas de ruptura excederam os valores calculados conforme as recomendações de norma. Já as vigas sem estribos romperam por cisalhamento com cargas de ruptura menor ou igual ao recomendado por norma. Este estudo mostra que não devem ser utilizadas vigas de alvenaria sem estribos.

ABSTRACT: An experimental study of the flexural behavior of concrete masonry beams subjected to flexure was done and the variables used were the height of the cross section and the distribution of the longitudinal reinforcement along the layers of blocks. Nine concrete masonry beams were cast and tested with concrete blocks of $13 \mathrm{MPa}$ compressive strength. The height of the cross section was defined by the number of blocks: one, two or three layers. The steel longitudinal reinforcement was distributed along the cross section, such that the steel arrangement gave the same steel reinforcing ratio for all beams. Three beams with lintels only and without stirrups were cast since its use if very common in the region of Goiania, GO. Results obtained were compared to those values obtained using recommendations prescribed in Brazilian code of practice NBR 15961-1 (2011). In general, beams with stirrups and with longitudinal reinforcement distributed along the height of the cross section had better performance, were more ductile and safer than beams without stirrups. These beams failed in flexure and ultimate loads were above those calculated using code recommendations. Beams without stirrups had a shear failure with ultimate loads lower than recommended by the code. This study shows that beams without stirrups should not be used.

\footnotetext{
* Contato com o autor:

${ }^{1}$ e-mail: mariaengenharia@gmail.com (M.G.D. Oliveira)

Mestre em Engenharia pela Universidade Federal de Goiás

2e-mail: bacarjievi@gmail.com (E. Bacarji)

Professor Doutor, Associado da Escola de Engenharia Civil da Universidade Federal de Goiás

${ }^{3}$ e-mail: rgilson.natal@gmail.com (G.N. Guimarães)

Professor Doutor, Titular da Escola de Engenharia Civil da Universidade Federal de Goiás (telefone 62+992219218)
} 


\section{INTRODUÇÃO}

O projeto de vigas de alvenaria estrutural pode ser elaborado utilizando o dimensionamento pelo estado limite último, semelhante ao utilizado para vigas de concreto armado. No entanto, a presença usual de furos nos blocos e a anisotropia da alvenaria gerada, principalmente, por juntas de argamassa que são planos de fragilidade, tornam o comportamento das vigas de alvenaria mais complexo que as de concreto armado. Apesar da literatura específica fornecer prescrições para o projeto de vigas de alvenaria, através da aplicação de formulações clássicas semelhantes às usadas no concreto armado, é limitada a informação experimental sobre os mecanismos resistentes que caracterizam o comportamento de vigas de alvenaria submetidas a esforços de cisalhamento e flexão (ALTRAN, 2010; LANDINI, 2001; HAACH, 2009; HAACH, 2011). Nesse contexto, esse estudo experimental do mecanismo resistente de vigas de alvenaria de canaletas e blocos de concreto vem fornecer contribuição para o comportamento de vigas de alvenaria estrutural e possibilita preencher lacunas na literatura específica.

$\mathrm{O}$ presente trabalho pretende analisar o comportamento de vigas de blocos e canaletas de concreto de alvenaria estrutural, submetidas à flexão simples e ensaiadas à ruptura com duas cargas concentradas simétricas em relação ao centro da viga posicionadas a um quarto do vão. Nove vigas de alvenaria de concreto foram ensaiadas contendo blocos com resistência à compressão de $13 \mathrm{MPa}$. A altura da seção transversal era definida pelo número de blocos ou canaletas podendo ser de uma, duas ou três fiadas. A armadura longitudinal de tração era distribuída ao longo da seção transversal de modo que a taxa de armadura permanecesse igual para todas as vigas. Três vigas foram moldadas sem estribos.

A geometria da seção transversal, o uso de blocos e canaletas e a distribuição da armadura longitudinal tiveram o objetivo de verificar como essas variáveis influenciariam as cargas e os modos de ruptura. Resultados dos ensaios foram comparados com valores correspondentes fornecidos pelas prescrições da norma brasileira de alvenaria estrutural NBR 15961-1 (ABNT, 2011)

Dentre as limitações deste trabalho podem ser considerados: uso de apenas um tipo de bloco com uma resistência de $13 \mathrm{MPa}$ e de um único fabricante, armadura de cisalhamento constante para todas as vigas com armadura de cisalhamento, ensaiadas apenas vigas bi-apoiadas, relação comprimento/altura da viga variando dentre 3,7 e 11,7 .

\subsection{DIMENSIONAMENTO DE VIGAS DE ALVENARIA ESTRUTURAL}

Segundo a norma brasileira de alvenaria estrutural NBR15961-1 (2011), as vigas de alvenaria armada são dimensionadas no estádio III, considerando que as tensões de tração na flexão não podem ser resistidas pela alvenaria, mas apenas pela armadura. As tensões de cisalhamento podem ser resistidas pela alvenaria ou pela armadura, isoladamente ou em conjunto.

A resistência de cálculo da alvenaria $\left(f_{d}\right)$ é obtida pela resistência característica dividida pelo coeficiente de ponderação das resistências $\left(\gamma_{\mathrm{m}}\right)$. Em geral, os valores de $\gamma_{m}$ para verificação no Estado Limite Último (ELU) em projeto indicados pela norma NBR 15961-2 (2011) são iguais a 2,0 para alvenaria e para o graute e igual a 1,15 para o aço.

O dimensionamento da armadura de tração em vigas de alvenaria é semelhante ao dimensionamento de armadura em vigas de concreto armado. Será apresentado resumo de algumas prescrições da norma e mais detalhes podem ser encontrados na NBR15961-1 (ABNT, 2011).

O concreto da seção transversal é considerado homogêneo mesmo sendo proveniente de potencialmente diferentes fontes como canaletas, blocos e graute. A expressão para a área de aço de tração As em vigas de alvenaria pode ser dada pela Equação 1.

$$
A_{s}=\frac{M}{f_{s}(d-0,4 x)}
$$

Em que:

$\mathbf{A}_{s}=$ área de aço de tração $\left(\mathrm{cm}^{2}\right)$;

$\mathbf{M}=$ o momento fletor de cálculo (kgf.cm);

$\mathbf{f}_{\mathrm{s}}=$ a tensão na armadura $\left(\mathrm{kgf} / \mathrm{cm}^{2}\right)$; 
$\mathbf{d}=$ altura útil $(\mathrm{cm})$;

$\mathbf{x}=$ a posição da linha neutra e é calculada com a expressão mostrada na Equação 2 (cm).

$$
x=\frac{A_{s} f_{s}}{0,8 \cdot f_{d} \cdot b}
$$

Em que:

$A_{s}=$ área de aço de tração $\left(\mathrm{cm}^{2}\right)$;

$\mathbf{f}_{\mathrm{s}}=$ a tensão na armadura $\left(\mathrm{kgf} / \mathrm{cm}^{2}\right)$ e considerada igual à metade da tensão de escoamento do aço;

$\mathbf{b}=$ largura da viga $(\mathrm{cm})$;

$\mathbf{f}_{\mathrm{d}}=$ a máxima tensão de compressão no concreto da alvenaria $\left(\mathrm{kgf} / \mathrm{cm}^{2}\right)$.

A resistência característica à compressão simples da alvenaria $f_{k}$ deve ser determinada com base no ensaio de paredes ou ser estimada como $70 \%$ da resistência característica de compressão simples de prisma $f_{p k}$ ou $85 \%$ da de pequena parede $f_{\text {ppk. }}$.

No dimensionamento ao cisalhamento, a norma NBR15961-1 (ABNT, 2011) considera a analogia de treliça onde uma parcela dos esforços de cisalhamento é resistida pela alvenaria e a outra pelos estribos transversais. Para peças de alvenaria estrutural submetidas à flexão e quando existirem armaduras perpendiculares ao plano do cisalhamento e envoltas por graute, a resistência característica ao cisalhamento $f_{\mathrm{vk}}$ pode ser obtida pela Equação 3.

$$
f_{v k}=0,35+17,5 \rho \leq 0,7 \mathrm{MPa}
$$

Onde:

$\mathbf{f}_{\mathrm{vk}}=\mathrm{a}$ resistência característica ao cisalhamento $\left(\mathrm{kgf} / \mathrm{cm}^{2}\right)$;

$\rho=$ a taxa geométrica de armadura.

Para a determinação das armaduras de cisalhamento pode-se descontar a parcela da força cortante absorvida pela alvenaria, $\mathrm{V}_{\mathrm{a}}$, dada pela Equação 4.

$$
v_{a}=f_{v d} \cdot b d
$$

\section{Em que:}

$\mathbf{V}_{\mathbf{a}}=$ força cortante absorvida pela alvenaria (kgf);

$\mathbf{f}_{\mathrm{vd}}=\mathrm{a}$ resistência de cálculo ao cisalhamento da alvenaria $\left(\mathrm{kgf} / \mathrm{cm}^{2}\right)$;

$\mathbf{b}=$ largura da seção transversal da viga $(\mathrm{cm})$;

$\mathbf{d}=$ altura útil seção transversal da viga $(\mathrm{cm})$.

A armadura de cisalhamento $A_{s w}$ é dada pela Equação 5.

$$
A_{s w}=\frac{\left(V_{d}-V_{a}\right) s}{0,5 \cdot f_{y d} \cdot d}
$$

Em que:

$\mathbf{A}_{\text {sw }}=$ armadura de cisalhamento $\left(\mathrm{cm}^{2}\right)$;

$\mathbf{V}_{\mathbf{d}}=$ força cortante de cálculo (kgf);

$V_{a}=$ força cortante absorvida pela alvenaria (kgf);

$\mathbf{f}_{\mathbf{y d}}=\mathbf{a}$ tensão de escoamento na armadura

$\left(\mathrm{kgf} / \mathrm{cm}^{2}\right)$;

$\mathbf{s}=$ espaçamento dos estribos $(\mathrm{cm})$;

$\mathbf{d}=$ altura útil da seção transversal da viga $(\mathrm{cm})$.

As pesquisas experimentais (LANDINI, 2001; MOHAMAD, 2007; OLIVEIRA, 2015) realizadas sobre vigas de alvenaria de concreto não são tão abundantes quanto em outras áreas da alvenaria estrutural, mas, dentre as mais recentes, aquelas desenvolvidas por HAACH (2009) se destacam. Seu programa experimental teve o objetivo de avaliar o comportamento à flexão e ao cisalhamento de vigas de alvenaria reforçadas e testadas à flexão, e foram executadas vinte e quatro vigas de alvenaria com diferentes geometrias, distribuição de unidades, e armadura horizontal. Podem-se citar suas principais conclusões: vigas de alvenaria sem armadura apresentam resistência à flexão muito reduzida e ruptura muito frágil; a armadura horizontal claramente aumentou a sua resistência à tração, melhorou a ductilidade através de notável aumento em sua capacidade de deformação; armadura vertical aumenta a resistência ao cisalhamento e proporciona uma melhor distribuição das fissuras; vigas de alvenaria construídas com enchimento das juntas verticais apresentaram maior carga de ruptura que as construídas sem enchimento das juntas verticais. 


\section{PROGRAMA EXPERIMENTAL}

Foram ensaiadas 9 vigas compostas de blocos e canaletas de concreto com resistência característica a compressão de $10 \mathrm{MPa}$ consolidados por juntas amarradas com argamassa com espessura de um centímetro. As vigas foram ensaiadas como bi-apoiadas sujeitas a duas cargas concentradas a $1 / 4$ e $3 / 4$ do vão conforme mostra o esquema de ensaio da Figura 1.

O comprimento das vigas era de $239 \mathrm{~cm}$, o que corresponde a 6 (seis) blocos de dimensões $14 \mathrm{~cm} \times 19 \mathrm{~cm} \times 39 \mathrm{~cm}$ mais 5 juntas com espessura de $1 \mathrm{~cm}$. O vão livre entre os apoios é de $219 \mathrm{~cm}$. A Figura 2 mostra a seção transversal e a armadura das vigas. A primeira fiada sempre é composta por blocos tipo canaleta de altura $19 \mathrm{~cm}$. Assim, as vigas com uma fiada possuíam altura de $19 \mathrm{~cm}$, com duas fiadas a altura era de $39 \mathrm{~cm}$ e com 3 fiadas a altura era de $59 \mathrm{~cm}$. A nomenclatura das vigas foi adotada visando evidenciar a geometria e forma de distribuição da armadura em cada situação a ser analisada. As vigas denominadas por VxC-y possuíam estribos verticais e eram constituídas por canaletas na primeira fiada e blocos nas demais fiadas, onde $x$ é o número de fiadas e y é o número de fiadas com armadura longitudinal. As vigas denominadas por VxCC-y eram constituídas apenas por canaletas em todas as fiadas e não possuíam estribos verticais. Essas vigas VxCC-y não possuíam armadura de cisalhamento pois as canaletas impedem a colocação de estribos verticais, mas esse tipo de viga é utilizada por construtores na região de Goiânia, GO. Todas as vigas com estribos possuíam a mesma quantidade de estribos e sua largura era de $14 \mathrm{~cm}$.

A prática convencional das vigas de concreto armado é concentrar a armadura de flexão em duas regiões, uma na parte superior e outra na parte inferior da viga. Essa prática produz o braço de alavanca máximo possível para o aço. No caso das vigas de alvenaria esta prática pode resultar em congestionamento das barras horizontais nas camadas extremas, pois, devido à espessura das paredes dos blocos, há uma limitada região interna para colocação das mesmas, o que se agrava ainda mais quando se faz necessária a colocação da armadura de cisalhamento.

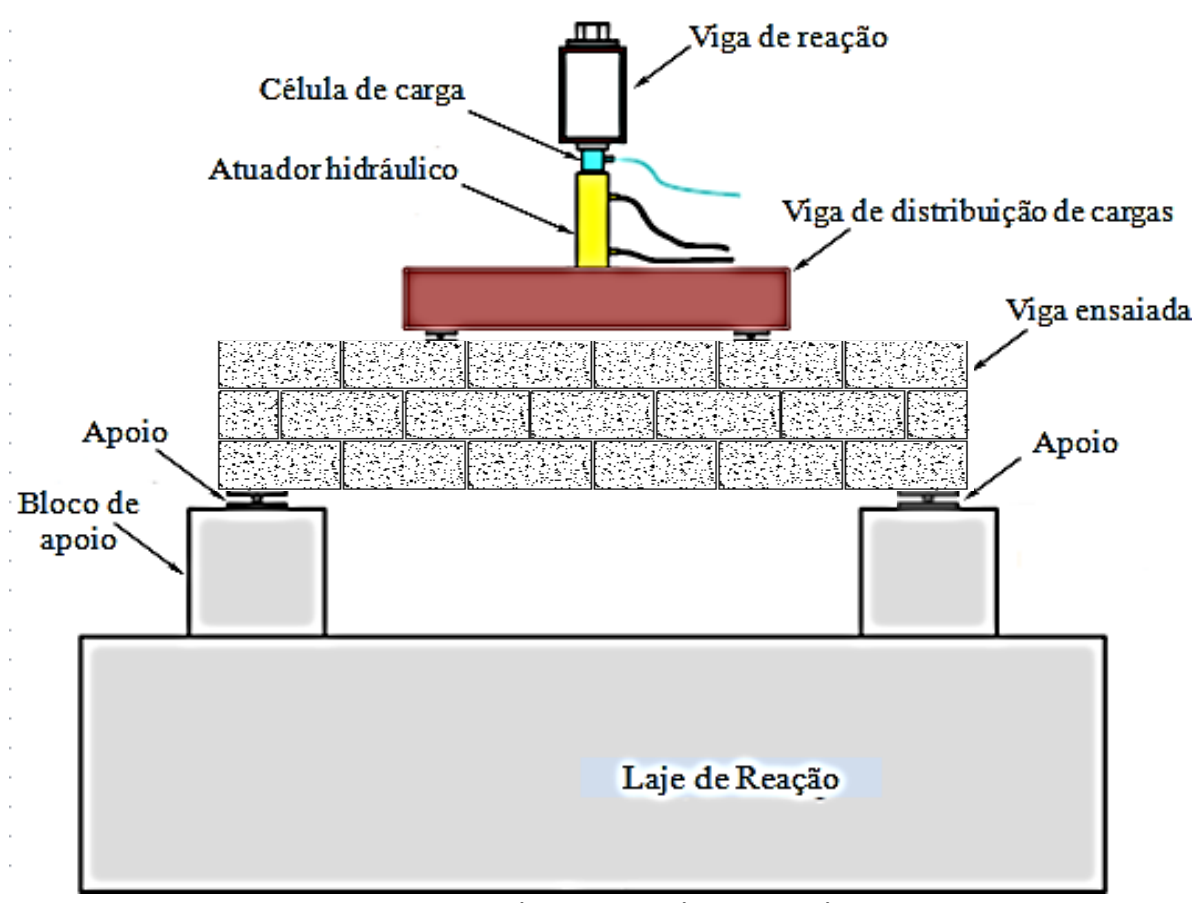

FIGURA 1: Esquema do aparato de ensaio das vigas.

FONTE: Os Autores. 

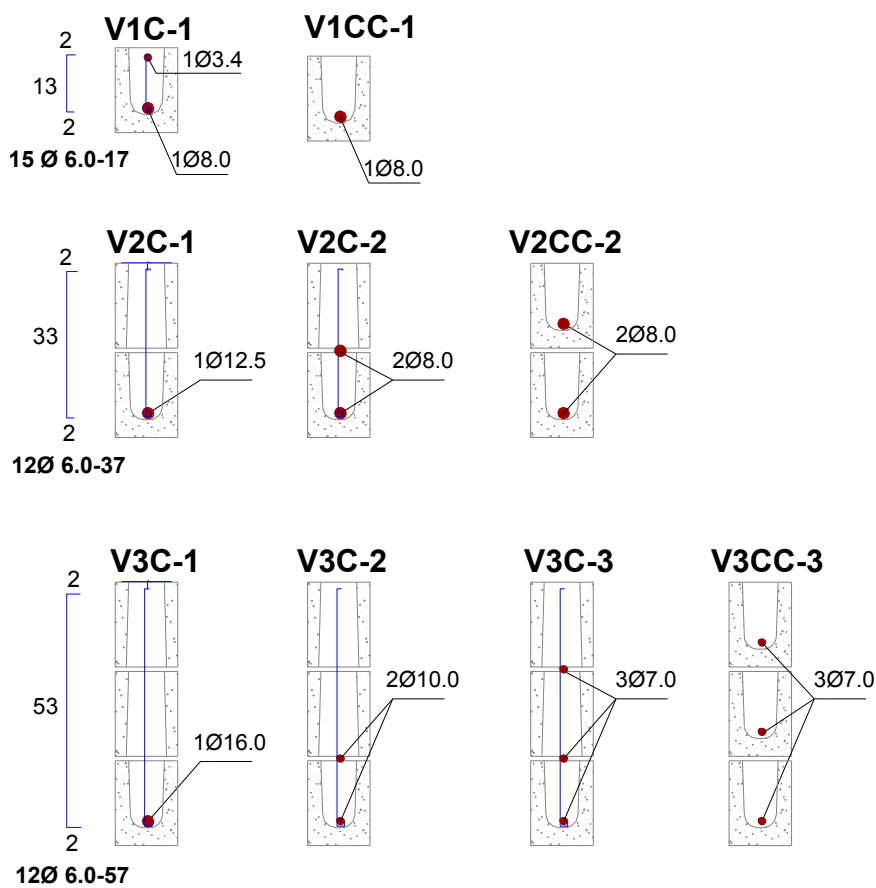

FIGURA 2: Detalhe da armadura na seção transversal das vigas. FONTE: Os Autores.

Com o intuito de analisar o efeito da distribuição das barras ao longo da altura das vigas, foram moldadas vigas com a armadura de flexão concentrada na primeira fiada e vigas com barras horizontais distribuídas ao longo das camadas de blocos, mantendo fixa a taxa da armadura de flexão em todas a vigas.

Os blocos e canaletas foram fabricados na Concrefato - Indústria de Artefatos de Concreto Ltda., localizada em Goiânia, GO e os ensaios de caracterização foram realizados no Laboratório de Materiais de Construção da Escola de Engenharia Civil da Universidade Federal de Goiás (UFG). Todos os ensaios seguiram os procedimentos preconizados pela norma brasileira NBR 12118 (ABNT, 2010), sendo eles: determinação da área líquida, teor de umidade, absorção e resistência à compressão axial dos blocos.

O graute foi dosado de forma a atingir, na idade do ensaio das vigas, a resistência à compressão de $20 \mathrm{MPa}$. A resistência à compressão da argamassa utilizada na união dos blocos foi fixada em $7 \mathrm{MPa}$. Na ocasião da confecção das vigas de alvenaria, foram moldados 9 (nove) prismas não grauteados e 9 (nove) prismas grauteados para rompimento em diferentes idades. A execução dos prismas de blocos de concreto foi realizada segundo os procedimentos preconizados pela NBR 15961-2 (ABNT, 2011) - Anexo A, para preparo e ensaio à compressão dos mesmos.

As vigas foram executadas e ensaiadas no Laboratório de Estruturas da Escola de Engenharia Civil da UFG. Visando não introduzir parâmetros desconhecidos, oriundos da divergência de mão de obra, todos os prismas e todas as vigas foram montadas pelo mesmo profissional, o qual possuía experiência em execução de alvenaria estrutural. No dimensionamento das armaduras utilizaram-se as prescrições da NBR 15961-2 (ABNT, 2011). A armadura de cisalhamento foi dimensionada de forma a não permitir ruptura por cisalhamento, e, por motivos executivos, o espaçamento dos estribos foi adequado à disposição dos furos dos blocos componentes da viga de alvenaria. Foram colocados estribos de bitola $6 \mathrm{~mm}$ espaçados a cada $19 \mathrm{~cm}$ (com alguns ajustes nos espaçamentos nas extremidades das vigas). Foi utilizado um pequeno gancho de aço, que era posicionado horizontalmente, para a fixação dos estribos para que estes não se movimentassem durante 0 grauteamento da viga.

Devido à imposição inicial de fixação da taxa da armadura horizontal (rh), as barras foram arranjadas, em bitolas e quantidades, conforme 
apresentado na Tabela 1. A Tabela 1 também mostra outras características das armaduras utilizadas. O aço utilizado para a armadura longitudinal foi CA-60: bitola de 7,0 mm e CA-50: bitolas de 8, 10, 12.5 e $16 \mathrm{~mm}$. Para a armadura transversal foi utilizado o aço CA-60 com bitola de 6 $\mathrm{mm}$.

Foram utilizados extensômetros elétricos de resistência (strain gages) da marca EXCEL, tipo PA-06-250BA-120-L nas barras da armadura horizontal e transversal e colocados conforme apresentado na Figura 3. Nas barras verticais, os extensômetros foram colados à meia altura.

Foram utilizados extensômetros elétricos de resistência no ponto médio das faces superiores da região central das vigas para medição de deformações no concreto.

Para medir os deslocamentos horizontais e verticais, foram instalados relógios comparadores digitais e LVDTs ao longo da viga nas suas faces inferior e laterais. Os relógios comparadores foram instalados na face inferior de viga para medição de flechas a $L / 4, L / 2$ e $3 / 4 L$, onde $L$ é o comprimento do vão livre. Os LVDTs foram instalados nas duas extremidades das vigas para medição dos deslocamentos horizontais de todas as fiadas. Foi também instalado um LVDT na face superior da região central da viga para medição da flecha no centro do vão.

O processo de montagem do ensaio das vigas obedeceu às seguintes etapas: posicionamento da viga; instalação dos aparelhos de apoio; posicionamento da célula de carga; verificação do prumo; marcação e posicionamento dos aparelhos medidores. Foi desenvolvido um sistema de transporte das vigas no laboratório, utilizando formas metálicas, para que estas não ficassem sujeitas a esforços (incluindo o peso próprio) antes de serem posicionadas no esquema de ensaio. As vigas foram ensaiadas aos 45 dias de idade.

\begin{tabular}{ccccccccccc}
\hline \multicolumn{7}{c}{ TABELA 1: Propriedades físicas e geométricas das armaduras das vigas. } \\
Viga & $\mathbf{d}(\mathbf{c m})$ & $\mathbf{d}^{\prime}(\mathbf{c m})$ & $\phi(\mathbf{m m})$ & $\mathbf{N} \phi$ & $\mathbf{A}_{\mathbf{s h}}\left(\mathbf{c m}^{2}\right)$ & $\boldsymbol{\rho}_{\mathbf{h}}(\mathbf{\%})$ & $\mathbf{x}(\mathbf{c m})$ & $\mathbf{K}_{\mathbf{x}}$ & $\mathbf{A}_{\mathbf{s w}}\left(\mathbf{c m}^{2}\right)$ \\
\hline V1C-1 & 0,145 & 0,045 & 8.0 & 1 & 0,503 & 0,248 & 1,16 & 0,080 & 1,49 \\
\hline V1CC-1 & 0,140 & 0,050 & 8.0 & 1 & 0,503 & 0,256 & 1,20 & 0,086 & 0,00 \\
\hline V2C-1 & 0,338 & 0,052 & 12.5 & 1 & 1,227 & 0,259 & 1,21 & 0,036 & 1,49 \\
\hline V2C-2 & 0,267 & 0,123 & 8.0 & 2 & 1,005 & 0,269 & 1,26 & 0,047 & 1,49 \\
\hline V2CC-2 & 0,267 & 0,123 & 8.0 & 2 & 1,005 & 0,269 & 1,26 & 0,047 & 0,00 \\
\hline V3C-1 & 0,538 & 0,052 & 16.0 & 1 & 2,011 & 0,267 & 1,25 & 0,026 & 1,49 \\
\hline V3C-2 & 0,467 & 0,123 & 10.0 & 2 & 1,571 & 0,24 & 1,12 & 0,024 & 1,49 \\
\hline V3C-3 & 0,333 & 0,257 & 7.0 & 3 & 1,155 & 0,248 & 1,50 & 0,045 & 1,49 \\
\hline V3CC-3 & 0,333 & 0,257 & 7.0 & 3 & 1,155 & 0,248 & 1,50 & 0,045 & 0,00 \\
\hline
\end{tabular}

FONTE: Os Autores.

Sendo:

$\mathrm{d}=$ Altura útil da seção transversal $(\mathrm{m})$;

$d^{\prime}$ = Distância entre o centro de gravidade da armadura longitudinal à borda mais comprimida $(m)$;

$\phi=$ Diâmetro da armadura horizontal $(\mathrm{mm})$;

$\mathrm{N} \phi=$ Quantidade de barras da armadura horizontal;

$A_{s h}=$ Área de aço da armadura horizontal $\left(\mathrm{cm}^{2}\right)$;

$\rho_{\mathrm{h}}=$ Taxa da armadura horizontal ( $\left.A_{s} / \mathrm{bh}\right)$, sendo $\mathrm{b}$ a dimensão da base da seção transversal da viga (\%);

$\mathrm{x}=$ Posição da linha neutra $(\mathrm{cm})$;

$\mathrm{K}_{\mathrm{x}}=$ Relação $\mathrm{x} / \mathrm{d}$;

$A_{s w}=$ Área de aço da armadura de cisalhamento $\left(\mathrm{cm}^{2}\right)$. 

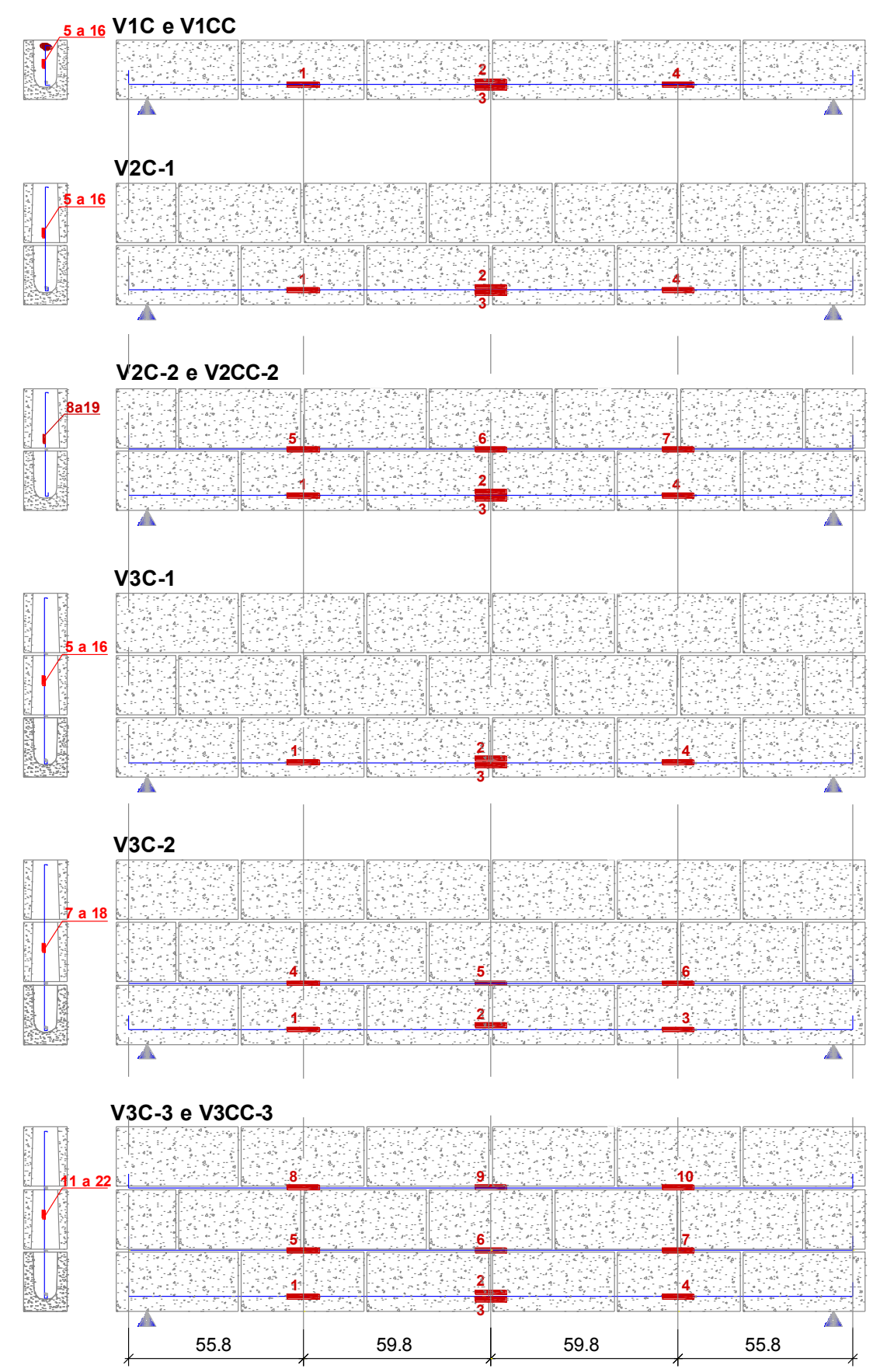

FIGURA 3: Posicionamento dos extensômetros nas barras da armadura FONTE: Os Autores

\section{RESULTADOS}

A seguir será feita a apresentação e análise dos principais resultados obtidos nos ensaios. Todas as vigas foram ensaiadas até a ruptura e armadura longitudinal entrou em escoamento na maioria das vigas, sendo que, em algumas peças, o ensaio foi prolongado até a ruptura completa da barra da armadura longitudinal localizada na primeira fiada.

A Figura 4 apresenta o gráfico da carga $x$ a flecha central até a carga de ruptura.

De modo geral, as curvas da Figura 4 mostram os três estágios de carregamento indicados na literatura tradicional: estágio préfissuração com grandes inclinações da curva, estágio pós-fissuração com uma menor inclinação que o estágio anterior e estágio pós-escoamento do aço onde a curva fica mais horizontalizada com baixa inclinação. As curvas das vigas V2C-1 e V3C-3 não mostram o terceiro estágio. Provavelmente existiu o 
terceiro estágio para as vigas $\mathrm{V} 2 \mathrm{C}-1$ e $\mathrm{V} 3 \mathrm{C}-3$, mas a ruptura é rápida e pouco dúctil sendo mais difícil a aquisição dos dados na iminência da ruptura. Para as vigas com a mesma altura da seção transversal, os deslocamentos máximos medidos no meio do vão foram maiores em vigas com menor altura para um mesmo estágio de carregamento. Em relação ao deslocamento limite de serviço de $L_{0} / 300$ (onde $L_{o}$ é o comprimento do vão) prescrito pela NBR 15961-1 (ABNT, 2011), foi verificado que apenas as vigas V2C-1 e V3C-3 romperam antes de atingirem esse limite, que neste caso é igual a $7,3 \mathrm{~mm}$, pelos motivos mencionados anteriormente.

A Figura 5 apresenta os diagramas carga $x$ deformação obtidos dos valores registrados através dos extensômetros instalados nas barras mais solicitadas da armadura longitudinal. A figura apresenta também os valores das deformações de escoamento $\varepsilon_{y}$ obtidas nos ensaios de tração dos aços. As deformações das barras da armadura longitudinal ultrapassaram a deformação de escoamento da maioria das vigas, exceção das vigas V3C-3 e V3CC-3. Nas vigas V1C-1, V2C-2, V2CC-2 e V3C-2 verificou-se visualmente a ruptura da barra de aço mais tracionada. A curva de deformação da armadura longitudinal da viga V3CC-3 teve um comportamento atípico se comparada com as demais, pois no início do carregamento até a carga de aproximadamente $52 \mathrm{kN}$, a deformação indica que a barra foi comprimida, e a partir daí foi tracionada; o que é incoerente devido a região da viga e posição da barra em questão. Aparentemente, o extensômetro pode não ter funcionado adequadamente. Na viga V3CC-3 houve desmembramento dos blocos provocando ruína da viga antes das barras de aço entrarem em escoamento (vide Figura 6d). Nessa viga, a última flecha medida foi menor que a flecha de serviço (vide Figura 4), que, junto com a falta de escoamento das barras aço, caracterizam uma ruptura frágil.

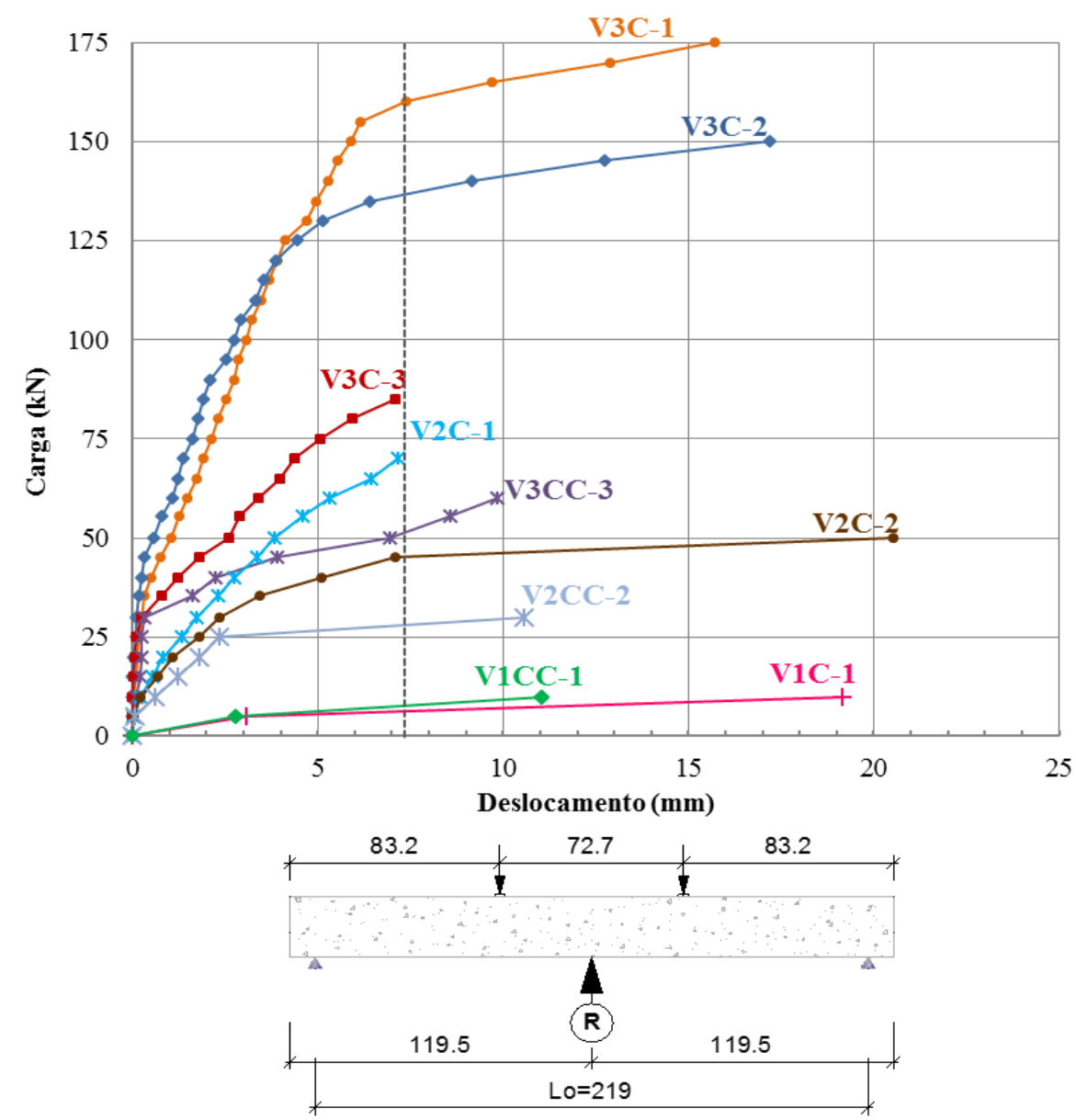

FIGURA 4: Curvas carga $x$ deslocamentos centrais. FONTE: Os Autores. 


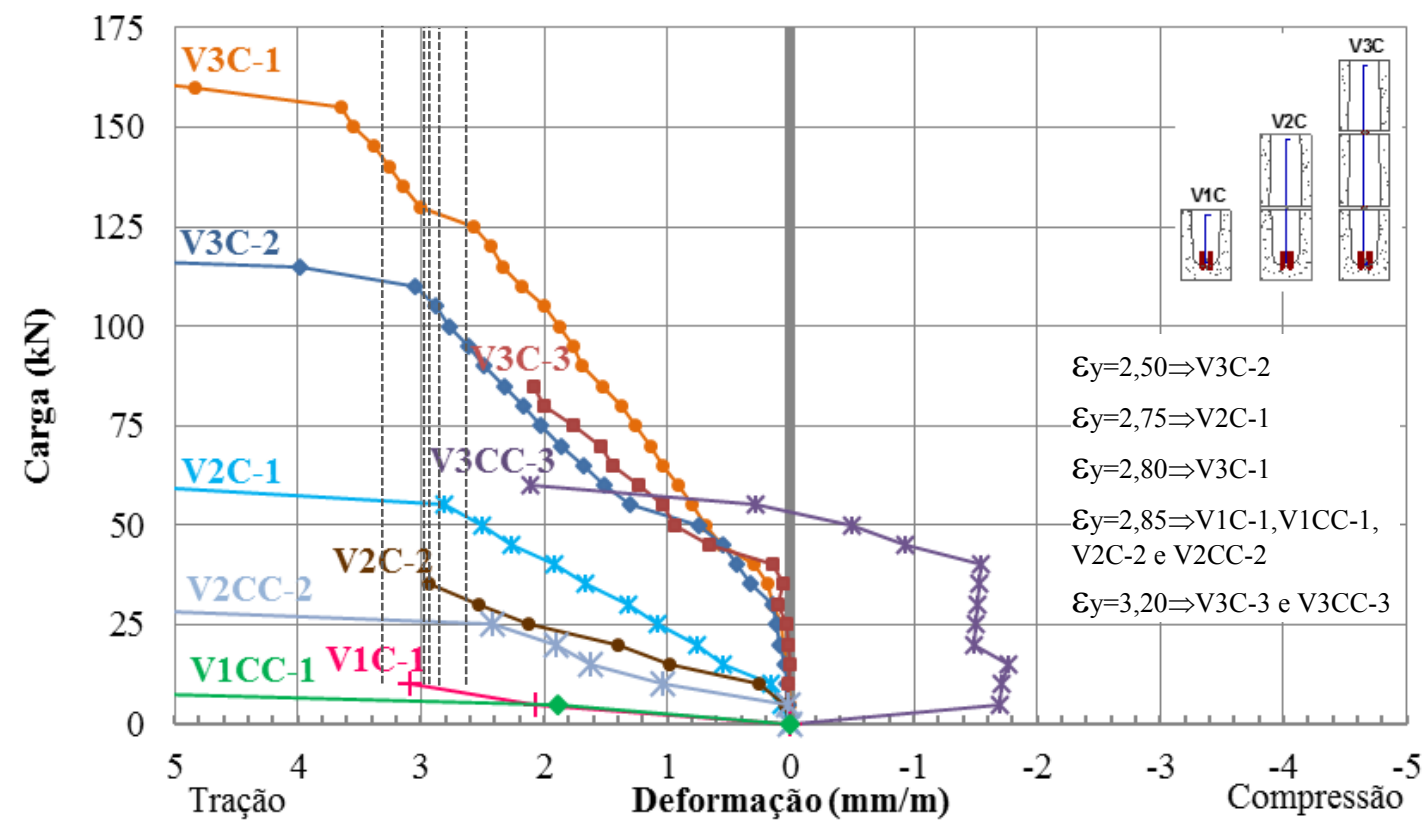

FIGURA 5: Diagrama carga x deformação das armaduras longitudinais na região mais tracionada da viga.

FONTE: Os Autores.

Já nas armaduras transversais (estribos), as deformações finais dos extensômetros não ultrapassaram o limite de escoamento para todas as vigas. A armadura transversal foi pouco exigida e a média das deformações máximas da armadura de cisalhamento foi de $0,22 \mathrm{~mm} / \mathrm{m}$, enquanto na armadura longitudinal esta média foi maior que 2 $\mathrm{mm} / \mathrm{m}$.

Para a análise das deformações do concreto foi considerada a deformação registrada pelos extensômetros instalados na região mais comprimida na face superior central da viga. Quanto à deformação última de ruptura do concreto, foi tomada como referência a especificada pelo NBR 15961-1 (ABNT, 2011) que é igual a $3,5 \mathrm{~mm} / \mathrm{m}$ para peças submetidas a flexão. As deformações máximas no concreto na região comprimida ficaram sempre abaixo de $3,5 \mathrm{~mm} / \mathrm{m}$, exceto para a viga V3CC-3 que atingiu $5,62 \mathrm{~mm} / \mathrm{m}$, mas neste caso essa leitura ocorreu com a viga já rompida. Em geral, a deformação máxima ficou entre 1,0 e $2,5 \mathrm{~mm} / \mathrm{m}$.

\subsection{FISSURAÇÃO, CARGA E MODO DE RUPTURA}

A Tabela 2 apresenta características principais das vigas, as cargas de primeira fissuração ( $\left.P_{\text {fiss }}\right)$, as cargas de escoamento $P_{y}$ (início do escoamento do aço segundo as leituras dos extensômetros na armadura longitudinal) e as cargas de ruptura $\mathrm{P}_{\mathrm{u}}$. A tabela mostra ainda a relação $R_{\text {fiss }}$ que é a relação entre a carga de fissuração e a carga de ruptura e a relação de ductilidade $R_{\text {duct }}$ que é a razão da carga de ruptura e a carga de escoamento que é um indicativo da ductilidade da viga. A última coluna da tabela indica o provável modo de ruptura. A altura útil mostrada nessa tabela é diferente mesmo em vigas com mesma altura, pois as bitolas, distribuição na seção transversal e quantidades de barras são diferentes. Como as armaduras das vigas V3C-3 e V3CC-3 ficaram na iminência de entrar em escoamento, foram consideradas iguais as cargas de escoamento e ruptura para efeitos desta tabela.

A Tabela 2 mostra que, como esperado, a carga de ruptura aumentou com o acréscimo de altura da viga. As maiores cargas de ruptura e de escoamento ocorreram nas vigas com uma barra na canaleta e com armadura de cisalhamento (V1C-1, V2C-1 e V3C-1) comparando com seus pares. A distribuição das barras longitudinais na seção transversal das vigas V2C-2, V2CC-2, V3C-2, V3C-3 e V3CC-3 reduziu a altura útil e, consequentemente, a carga de ruptura e de fissuração. 
TABELA 2: Cargas e modos de ruptura das vigas.

\begin{tabular}{|c|c|c|c|c|c|c|c|c|c|}
\hline Viga & $\begin{array}{l}\rho \\
(\%)\end{array}$ & $\begin{array}{c}\mathrm{H} \\
(\mathrm{cm} \\
1\end{array}$ & $\begin{array}{c}D \\
(\mathrm{~cm})\end{array}$ & $\begin{array}{l}P_{\text {fiss }} \\
(k N)\end{array}$ & $\begin{array}{c}\mathrm{P}_{\mathrm{y}} \\
(\mathrm{kN})\end{array}$ & $\begin{array}{c}\mathrm{P}_{\mathrm{u}} \\
(\mathrm{kN})\end{array}$ & $R_{\text {fiss }}$ & $\mathrm{R}_{\text {Duct }}$ & $\begin{array}{c}\text { Provável Modo } \\
\text { de Ruptura }\end{array}$ \\
\hline V1C-1 & $\begin{array}{c}0,2 \\
5 \\
\end{array}$ & 19 & 15 & 4,0 & 7,4 & 10,7 & 0,37 & 1,46 & EA-Ruptura Aço \\
\hline V1CC-1 & $\begin{array}{c}0,2 \\
6\end{array}$ & 19 & 14 & 3,8 & 7,5 & 10,5 & 0,37 & 1,40 & EA-Cisalhamento \\
\hline V2C-1 & $\begin{array}{c}0,2 \\
6 \\
\end{array}$ & 39 & 34 & 23,7 & 55,0 & 68,0 & 0,35 & 1,24 & EA-Ruptura Aço \\
\hline V2C-2 & $\begin{array}{c}0,2 \\
7 \\
\end{array}$ & 39 & 27 & 15,2 & 33,0 & 52,0 & 0,29 & 1,58 & EA-Ruptura Aço \\
\hline V2CC-2 & $\begin{array}{c}0,2 \\
7 \\
\end{array}$ & 39 & 27 & 15,2 & 26,0 & 29,0 & 0,52 & 1,12 & EA-Cisalhamento \\
\hline V3C-1 & $\begin{array}{c}0,2 \\
7 \\
\end{array}$ & 59 & 54 & 62,7 & 127,5 & 175,0 & 0,36 & 1,37 & EA \\
\hline V3C-2 & $\begin{array}{c}0,2 \\
4\end{array}$ & 59 & 47 & 42,5 & 90,0 & 150,0 & 0,28 & 1,67 & EA-Ruptura Aço \\
\hline V3C-3 & $\begin{array}{c}0,2 \\
5 \\
\end{array}$ & 59 & 33 & 26,3 & 84,0 & 84,0 & 0,31 & 1,00 & EA-Ruptura Aço \\
\hline V3CC-3 & $\begin{array}{c}0,2 \\
5 \\
\end{array}$ & 59 & 33 & 26,3 & 61,0 & 61,0 & 0,43 & 1,00 & EA-Cisalhamento \\
\hline
\end{tabular}

\section{Sendo:}

$\rho=$ taxa de armadura longitudinal de tração;

$\mathbf{h}=$ altura da seção transversal;

d = altura útil da seção transversal;

$\mathbf{P}_{\text {fiss }}=$ Carga de fissuração (sob a qual foi observada a primeira fissura);

$\mathbf{P}_{\mathbf{y}}=$ Carga de escoamento do aço;

$\mathbf{P}_{\mathbf{u}}=$ Carga de ruptura obtida no ensaio;

$\mathbf{R}_{\text {fiss }}=\mathrm{P}_{\text {fiss }} / \mathrm{P}_{\mathrm{u}}$ - Relação entre a carga de fissuração e a carga de ruptura;

$\mathbf{R}_{\text {Duct }}=\mathrm{P}_{\mathrm{u}} / \mathrm{P}_{\mathrm{y}}$ - Relação entre a carga de ruptura e a carga de escoamento;

EA-Ruptura aço = Escoamento da armadura de flexão (horizontal) com posterior ruptura da barra de aço;

EA-Cisalhamento = Ruptura por cisalhamento com escoamento da armadura de flexão (horizontal).

Quanto as vigas V1CC-1, V2CC-2 e V3CC-3 constituídas somente de blocos canaleta e com armadura de flexão distribuída na seção transversal e sem armadura de cisalhamento, os dados da Tabela 2 mostram que as mesmas sofreram redução na carga de ruptura e redução de ductilidade (menor relação $R_{\text {duct }}$ ) e aumento no parâmetro $R_{\text {fiss, }}$ relativamente aos seus pares com armadura de cisalhamento. Em relação a fissuração, podemos observar que as vigas V2CC-2 e V3CC-3 tiveram as maiores relações $\mathrm{R}_{\text {fiss }}$ e cerca de $30 \%$ maior que a média de todas as vigas que é de 0,37. Essas vigas sem armadura de cisalhamento apresentaram um panorama de evolução das fissuras com as mesmas distribuindo-se pela junta de argamassa com consequente desmembramento dos blocos, o que fragilizou rapidamente o bloco de compressão da viga levando a uma ruptura menos dúctil, isto é, sem grandes deformações.

Todas as vigas, exceto as vigas $\mathrm{V} 3 \mathrm{C}-3$ e V3CC-3, podem ser consideradas dúcteis pois a relação $R_{\text {duct }}$ é maior que a unidade e todas romperam após o escoamento do aço. Em média, a relação $R_{\text {duct }}$ é igual a 1,32 , o que é considerado relativamente baixa ductilidade se comparado a estruturas de aço ou concreto armado. Como a carga de escoamento $P_{y}$ é obtida a partir da leitura dos extensômetros na armadura mais tracionada, o seu valor depende do funcionamento do extensômetro. A Figura 6 indica que a armadura longitudinal das vigas V3C-3 e V3CC-3 não escoaram. A Figura 6(d) mostra a fotografia da viga V3CC-3 indica que sua armadura longitudinal se rompeu completamente.

Comparando as vigas com presença de armadura vertical de cisalhamento V2C-1 com V2C- 
2 e V3C-1 com V3C-2 com V3C-3, os dados da Tabela 2 mostram que o aumento no número de camadas de barras horizontais faz aumentar a ductilidade medida pelo parâmetro $R_{\text {duct }}$, mas ao mesmo tempo diminui a carga de ruptura pois a altura útil diminui. Comparando as vigas com presença de armadura vertical de cisalhamento V1C-1 com V2C-1 com V3C1 e V2C-2 com V3C-2, os dados da Tabela 2 mostram que o aumento no número de fiadas, sem distribuir a armadura longitudinal para as outras camadas, praticamente não tem alteração na ductilidade medida pelo parâmetro $R_{\text {duct }}$ mas, evidentemente, aumenta a carga de ruptura pois a altura útil aumenta.

A seguir, na Figura 6, são mostradas fotografias das vigas de 3 fiadas na sua configuração de ruptura. As outras vigas não são mostradas por limitações de espaço editorial.

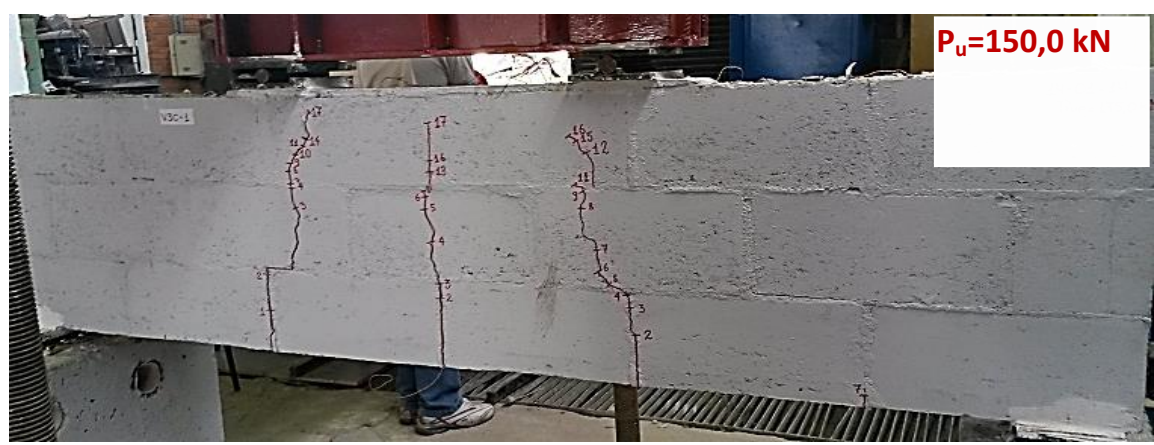

(a) Viga V3C-1

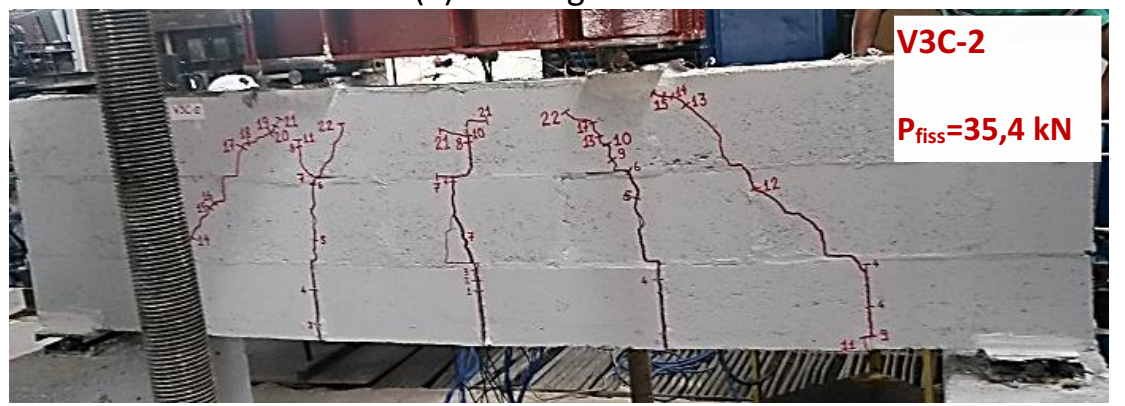

(b) Viga V2C-2

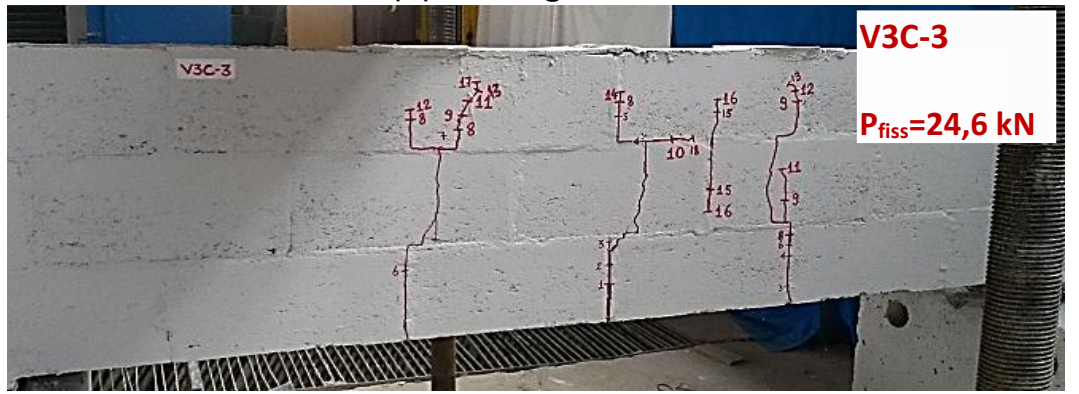

(c) Viga V3C-3

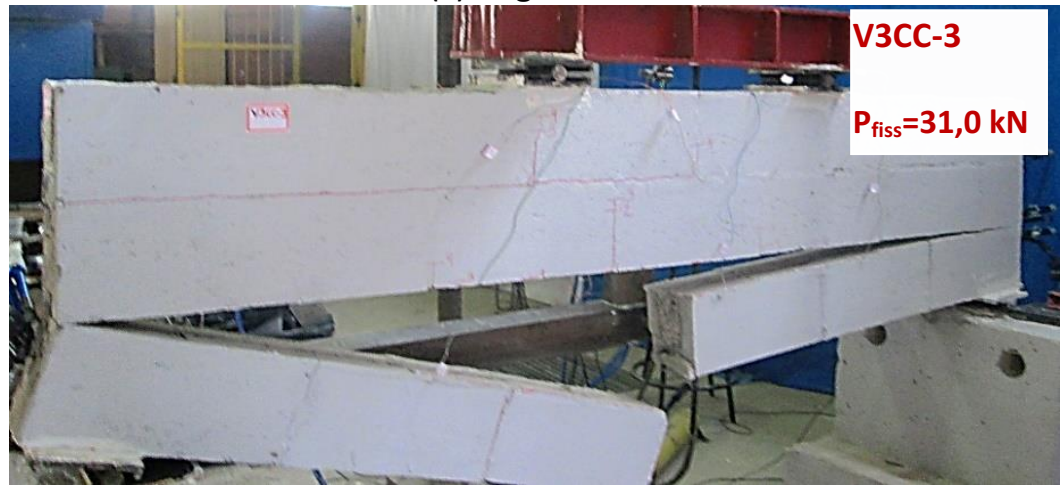

(d) Viga V3CC-3

FIGURA 6: Fotografias das vigas de 3 fiadas na configuração de ruptura.

FONTE: Os Autores. 


\subsection{COMPARATIVO ENTRE RESULTADOS EXPERIMENTAIS E OBTIDOS COM A NORMA NBR 15961-1}

A Tabela 3 apresenta a comparação entre a carga de ruptura obtida experimentalmente e a determinada através das prescrições da NBR 159611 (ABNT, 2011). Na Tabela 3, a carga de ruptura $\mathrm{P}_{\mathrm{u}}{ }^{k}$ foi calculada segundo as prescrições da norma, mas utilizando todos os coeficientes de segurança iguais à unidade e utilizando os valores característicos obtidos nos ensaios dos materiais. Esta carga de ruptura $\mathrm{P}_{\mathrm{u}}{ }^{\mathrm{k}}$ é aqui denominada por carga de ruptura característica. Já a carga de ruptura $\mathrm{P}_{\mathrm{u}}{ }^{\mathrm{d}}$ foi obtida seguindo todas as prescrições das normas com os coeficientes de segurança prescritos e os valores característicos dos materiais especificados em norma, e seria comparável à carga que seria obtida num escritório de projetos. Esta carga de ruptura $\mathrm{P}_{\mathrm{u}}{ }^{\mathrm{d}}$ é aqui denominada por carga de ruptura de projeto. Comparando a carga de ruptura característica $\mathrm{P}_{\mathrm{u}}{ }^{\mathrm{k}}$ com as cargas de ruptura obtidas nos ensaios $P_{u}$, todas as vigas ultrapassaram a sua respectiva carga de ruptura característica teórica.

A Tabela 3 mostra que a carga de ruptura obtida nos ensaios $\mathrm{P}_{\mathrm{u}}$ foi, em média, $13 \%$ maior que a carga de ruptura característica $\mathbf{P}_{\mathbf{u}}{ }^{k}$ (relação $\mathbf{P}_{\mathbf{u}} / \mathbf{P}_{\mathbf{u}}{ }^{k}$ ). Considerando a relação entre as cargas de ruptura de ensaio e a carga de ruptura de projeto (relação $\left.\mathbf{P}_{\mathrm{u}} / \mathbf{P}_{\mathbf{u}}{ }^{d}\right)$, verificamos que a carga de ruptura de ensaio é cerca de 4 vezes a carga de ruptura de projeto. O desvio padrão entre os resultados obtidos para a relação $\mathbf{P}_{\mathbf{u}} / \mathbf{P}_{\mathbf{u}}{ }^{k}$ é de 0,22 e para a relação $P_{u} / P_{u}{ }^{d}$ é de 0,72 . Esta minoração da carga de projeto de cerca de 0,25 da carga experimental poderia indicar que as minorações da carga de ruptura de projeto da NBR 15961-1 (ABNT, 2011) seriam excessivas quando consideramos como parâmetros os valores obtidos experimentalmente. Mas devido ao modo de ruptura da alvenaria ser caracterizado por pequenas deformações até próximo à ruptura (baixa ductilidade), elas são aceitáveis para vigas de alvenaria armada e compatíveis com fatores de segurança utilizados na alvenaria estrutural não armada.

TABELA 3: Comparação das cargas de ruptura experimentais e segundo a norma NBR 15961-1.

\begin{tabular}{|c|c|c|c|c|c|c|c|}
\hline VIGA & $\mathrm{L}_{\mathrm{o}} / \mathrm{h}$ & $\begin{array}{c}\mathbf{P}_{\mathrm{u}} \\
(\mathrm{kN})\end{array}$ & $\begin{array}{l}\mathbf{P}_{\mathrm{u}}{ }^{\mathrm{K}} \\
(\mathrm{kN})\end{array}$ & $\begin{array}{l}P_{u}^{d} \\
(k N)\end{array}$ & $\mathbf{P u}_{\mathrm{u}} / \mathbf{P u}^{\mathrm{k}}$ & $\mathbf{P u}_{\mathrm{u}} / \mathbf{P}_{\mathrm{u}}{ }^{\mathrm{d}}$ & $P_{u}{ }^{k} / P_{u}{ }^{d}$ \\
\hline V1C-1 & \multirow{2}{*}{11,53} & 10,7 & 10,0 & 2,9 & 1,07 & 3,74 & 3,51 \\
\hline $\mathrm{V} 1 \mathrm{CC}-1$ & & 10,5 & 9,7 & 2,7 & 1,09 & 3,82 & 3,52 \\
\hline $\mathrm{V} 2 \mathrm{C}-1$ & \multirow{3}{*}{6,13} & 68,0 & 58,2 & 17,0 & 1,17 & 4,01 & 3,43 \\
\hline $\mathrm{V} 2 \mathrm{C}-2$ & & 52,0 & 37,5 & 10,9 & 1,39 & 4,79 & 3,45 \\
\hline $\mathrm{V} 2 \mathrm{CC}-2$ & & 29,0 & 37,5 & 10,9 & 0,77 & 2,67 & 3,45 \\
\hline V3C-1 & \multirow{4}{*}{3,71} & 175,0 & 152,5 & 44,8 & 1,15 & 3,91 & 3,41 \\
\hline V3C-2 & & 150,0 & 103,4 & 30,3 & 1,45 & 4,95 & 3,41 \\
\hline V3C-3 & & 84,0 & 69,9 & 18,8 & 1,20 & 4,48 & 3,72 \\
\hline V3CC-3 & & 61,0 & 69,9 & 18,8 & 0,87 & 3,25 & 3,72 \\
\hline \multicolumn{5}{|r|}{ Média } & 1,13 & 3,96 & 3,51 \\
\hline & & & & Desvio Padrão & 0,22 & 0,72 & 0,12 \\
\hline
\end{tabular}

Onde:

$\mathbf{P}_{\mathbf{u}}=$ Carga de ruptura obtida no ensaio;

$\mathbf{P}_{\mathbf{u}}{ }^{k}{ }_{\text {NBR }} 15961-1=$ Carga de ruptura característica obtida segundo a norma NBR15961-1(2011) ;

$\mathbf{P}_{\mathrm{u}}{ }^{\mathrm{d}}$ NBR 15961-1 $=$ Carga de ruptura de projeto obtida segundo a norma NBR15961-1(2011) ;

$L_{0} / h=$ Relação entre o vão livre e a altura total da viga; 
De modo geral, as vigas com 2 ou 3 fiadas de blocos e com armadura vertical tiveram maiores relações $\mathrm{P}_{\mathrm{u}} / \mathrm{P}_{\mathrm{u}}{ }^{k}$ e $\mathrm{P}_{\mathrm{u}} / \mathrm{P}_{\mathrm{u}}{ }^{d}$ quando comparadas às vigas com somente uma fiada. Nas vigas com 2 ou 3 fiadas, mas sem armadura de cisalhamento, estas relações $\mathrm{P}_{\mathrm{u}} / \mathrm{P}_{\mathrm{u}}{ }^{k}$ e $\mathrm{P}_{\mathrm{u}} / \mathrm{P}_{\mathrm{u}}{ }^{d}$ diminuíram quando comparadas às vigas com armadura de cisalhamento. Sugere-se sempre utilizar estribos, pois sua ausência pode levar a menores cargas de rupturas.

A Tabela 4 apresenta a comparação entre os esforços cortante de ruptura obtidos experimentalmente e os determinados através das prescrições da NBR 15961-1 (ABNT, 2011). O esforço cortante de ruptura $\mathrm{V}_{\mathrm{u}}$ corresponde ao esforço cortante na barra no momento da ruptura e é igual à metade da carga de ruptura $\mathrm{P}_{\mathrm{u}}$. Analogamente às cargas de ruptura $\mathrm{Pu}_{\mathrm{u}}{ }^{k}$ e $\mathrm{Pu}_{\mathrm{u}}{ }^{\mathrm{d}}$ mostradas na Tabela 4, o esforço cortante de ruptura $\mathrm{V}_{\mathrm{u}}{ }^{\mathrm{k}} \mathrm{e} \mathrm{V}_{\mathrm{u}}{ }^{\mathrm{d}}$ correspondem ao esforço cortante calculado utilizando as prescrições da norma. $O$ esforço cortante $V_{u}{ }^{k}$ foi calculado utilizando coeficientes de segurança iguais a um e utilizando os valores característicos obtidos nos ensaios dos materiais e o esforço cortante $\mathrm{V}_{\mathrm{u}}{ }^{\mathrm{d}}$ corresponderia ao esforço cortante de ruptura de projeto.

TABELA 4: Comparação das cargas de cisalhamento experimentais e segundo a norma NBR 15961-1.

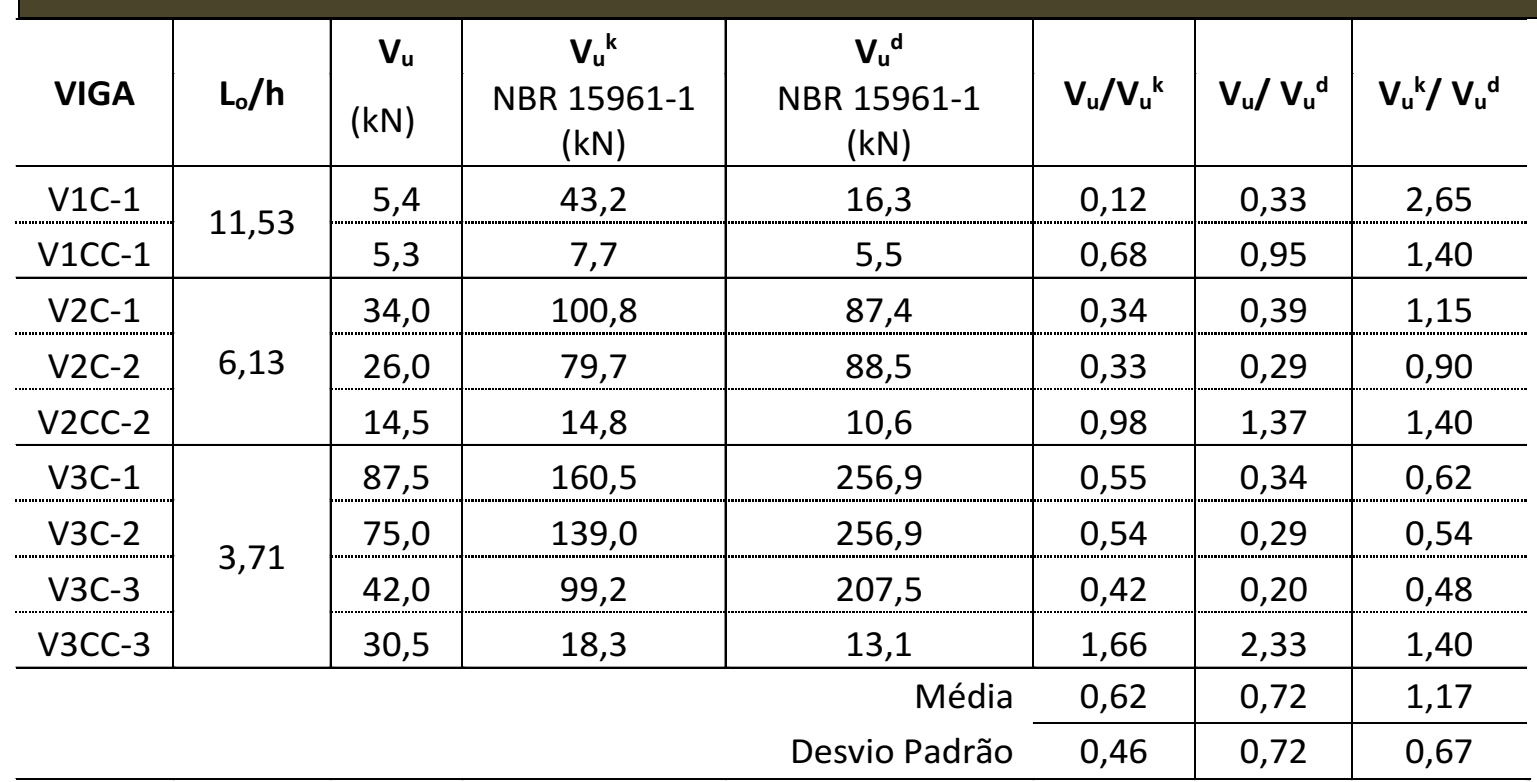

Onde:

$\mathbf{V u}=$ Carga de cisalhamento última obtida no ensaio;

$\mathbf{V u}^{\mathbf{k}}{ }_{\text {NBR 15961-1 }}$ Carga de cisalhamento última característica segundo a NBR15961-1(2011) ;

$\mathbf{V u}^{\mathrm{d}}{ }_{\text {NBR }}{ }_{15961-1}=$ Carga de cisalhamento última para projeto segundo a NBR15961-1(2011) ;

$\mathrm{L}_{\mathrm{o}} / \mathbf{h}=$ Relação entre vão livre e a altura total da viga.

Com a análise dos valores das cargas de cisalhamento na ruptura da viga com armadura de cisalhamento, apresentados na Tabela 4, observamos que nenhuma viga foi submetida a carga de cisalhamento maior que a sua carga de cisalhamento de ruptura, o que juntamente com os resultados registrados pelos extensômetros instalados nas barras da armadura de cisalhamento, todos inferiores ao limite de escoamento das barras, embasa a afirmação de que nenhuma viga com armadura vertical rompeu ao cisalhamento. $O$ valor médio da relação entre 0 esforço cortante experimental e o de projeto $\left(V_{u} / V_{u}{ }^{d}\right)$ foi de 0,31 para essas vigas. Quando essa análise é feita considerando todas as vigas, o valor médio da relação entre o esforço cortante experimental e o de projeto $\left(V_{u} / V_{u}{ }^{d}\right)$ sobe para 0,72 . Isto acontece, pois, os valores de $\mathrm{V}_{\mathrm{u}} / \mathrm{V}_{\mathrm{u}}{ }^{\mathrm{d}}$ aumentam substancialmente para as vigas sem armadura de cisalhamento (vigas V1CC-1, V2CC-2 e V3CC-3) e o valor médio da relação entre o esforço cortante experimental e o de projeto $\left(\mathrm{V}_{\mathrm{u}} / \mathrm{V}_{\mathrm{u}}{ }^{d}\right)$ para essas vigas 
é igual a 1,55. Essas vigas sem armadura vertical têm esforço cortante de ruptura $\mathrm{Vu}$ praticamente igual ou superior ao esforço cortante de projeto $\mathrm{V}_{\mathrm{u}}{ }^{\mathrm{d}}$. Como a ductilidade dessas vigas sem armadura de cisalhamento medida pelo parâmetro $R_{\text {duct }}$ (vide Tabela 2) foi sempre menor que as vigas correspondentes com armadura de cisalhamento, provavelmente ocorreu uma ruptura frágil por cisalhamento nas juntas dessas vigas seguido quase que instantaneamente por uma ruptura por tração na barra longitudinal da primeira fiada. Essa ruptura frágil e rápida foi mais difícil de ser detectada pela instrumentação. Aparentemente, as fissuras nas juntas de argamassa podem ter iniciado a ruptura mais frágil das vigas sem estribos por cisalhamento.

\section{CONCLUSÕES}

Considerando as limitações deste estudo, a seguir, são apresentadas as suas principais conclusões.

- A carga de ruptura aumentou com o acréscimo de altura da viga. Os maiores aumentos ocorreram nas vigas com barras somente na primeira fiada e com armadura de cisalhamento (V1C-1, V2C-1 e V3C-1). Em geral, os resultados obtidos indicam que a distribuição das barras na altura da seção transversal, apesar de reduzir a altura útil e consequentemente a carga de ruptura, melhorou o modo de ruptura através do aumento da ductilidade

- Todas as vigas com armadura de cisalhamento tiveram ruptura dúctil e a carga de ruptura é muito acima da carga de ruptura prevista em projeto (cerca de 4 vezes maiores), o que poderia indicar que as majorações para a carga de ruptura de projeto seriam excessivas, mas devido ao modo de ruptura da alvenaria ser caracterizado por pequenas deformações até próximo à ruptura (baixa ductilidade), essas majorações são aceitáveis para vigas de alvenaria armada. Já as vigas sem armadura de cisalhamento tiveram ruptura menos dúctil e o esforço cortante de ruptura foi maior ou igual ao esforço cortante de projeto indicando possível ruptura por cisalhamento.

- Verificou-se que a não inserção da armadura de cisalhamento reduz consideravelmente $\mathrm{o}$ momento resistente $\mathrm{e} a$ ductilidade das vigas. Nesse estudo todas as vigas detalhadas sem armadura de cisalhamento tiveram ruptura frágil indicando que pode ter havido ruptura por cisalhamento instantes antes da ruptura por tração da barra longitudinal de flexão. Assim, não se recomenda o uso de vigas de alvenaria sem armadura de cisalhamento.

\section{AGRADECIMENTOS}

Os autores agradecem à Coordenação de Aperfeiçoamento de Pessoal de Nível Superior CAPES e às empresas REALMIX Concreto Ltda. e Carlos Campos Consultorias Ltda. pelo apoio financeiro e suporte de laboratório dado ao desenvolvimento desta pesquisa.

\section{REFERÊNCIAS BIBLIOGRÁFICAS}

ASSOCIAÇÃO BRASILEIRA DE NORMAS TÉCNICAS, NBR 15961-1: Alvenaria Estrutural - Blocos de Concreto Parte 1: Projeto. Brasil, 2011.

NBR 15961-2: Alvenaria Estrutural - Blocos de Concreto Parte 2: Execução e controle de obras. Brasil, 2011.

NBR 12118: Blocos vazados de concreto simples para alvenaria - Métodos de ensaio, Brasil, 2010.

ALTRAN, D. A. Procedimento numérico para análise de vigas de alvenaria estrutural submetidas à flexão simples, 469p. - Dissertação (Mestrado em Engenharia Civil) - Universidade Estadual Paulista. Faculdade de Engenharia de llha Solteira, 2010.

HAACH, V. G. Development of a design method for reinforced masonry subjected to in-plane loading based on experimental and numerical analysis, PhD Thesis, University of Minho, Portugal, 2009.

HAACH, V. G.; VASCONCELOS G.; LOURENÇO P. B. Experimental analysis of reinforced concrete block masonry spandrels using pre-fabricated planar trussed bars. Construction Build Mater, 2011, in press [ doi:10.1016/j.conbuildmat.2011.06.005 ].

LANDINI, F. S. Comportamento à flexão e ao esforço cortante de vigas de alvenaria estrutural. $118 \mathrm{f}$. Dissertação (Mestrado)- Faculdade de Engenharia Civil, Universidade Estadual de Campinas, Campinas, 2001.

MOHAMAD, G. (2007) Mechanism failure of concrete 
block masonry under compression, $\mathrm{PhD}$ Thesis, University of Minho, Guimarães, Portugal, 2007. Disponível de www.civil.uminho.pt/masonry (em Português).

OLIVEIRA, M. G. D. Análise teórico-experimental de vigas de alvenaria estrutural submetidas à flexão simples. 142f. Dissertação (Mestrado)- Escola de Engenharia Civil, Universidade Federal de Goiás, Goiânia, GO, 2015. 